Bull. Austral. Math. Soc.

$46 \mathrm{~B} 20,46 \mathrm{~B} 03$

VOL. 65 (2002) [223-230]

\title{
WEAKLY COMPACT SETS AND SMOOTH NORMS IN BANACH SPACES
}

\author{
Marián Fabian, Vicente Montesinos and VáclaV Zizler
}

Two smoothness characterisations of weakly compact sets in Banach spaces are given.

One that involves pointwise lower semicontinuous norms and one that involves projectional resolutions of identity.

The Gâteaux smoothness of norms has a profound impact on the structure of nonseparable Banach spaces, especially if the smoothness is accompanied by additional properties like pointwise lower semicontinuity, lattice property or projectional resolutions of identity (see for example, $[3,4,5,6,7,8,9,10]$ ).

The purpose of the present note is to discuss the relationship between the Gâteaux smoothness of norms and the weak compactness of sets in Banach spaces. The result in Theorem 1 is of interest in separable spaces as well.

Let $M$ be a bounded set in a Banach space $(X,\|\cdot\|)$. We shall say that the norm $\|\cdot\|$ is $M$-smooth at $0 \neq x \in X$ if

$$
\sup \{\|x+t h\|+\|x-t h\|-2\|x\| ; h \in M\}=o(t) \text { for } t>0 .
$$

The norm is $M$-smooth if it is $M$-smooth at every point $0 \neq x \in X$.

If $M=B_{X}$, we get the usual notion of Fréchet differentiability (see for example [6]).

If $M$ is linearly dense in $X$ (that is, if $\overline{\operatorname{span}} M=X$ ), then $M$-smoothness implies the usual Gâteaux smoothness. If $X$ is a separable Banach space and its norm is Gâteaux smooth, then this norm is $M$-smooth for a linearly dense set $M \subset X$. Indeed, if $\left\{x_{i} ; i\right.$ $\in \mathbb{N}\}$ is a countable dense set in $B_{X}$, then $M:=\left\{i^{-1} x_{i} ; i \in \mathbb{N}\right\}$ works. Both these things can be seen by using the Lipschitz property of the norm.

An example of a Banach space $X$ with Gâteaux smooth norm that has no equivalent $M$-smooth norm for any linearly dense set $M$ in $X$ is a non weakly compactly generated subspace of a weakly compactly generated space of density $\omega_{1}$ (see [13] and Corollary 5 below if one assumes the Continuum Hypothesis).

Received 23rd July, 2001

The first and third named authors were supported by AV 1019003 and GAČR 201-01-1198 (Czech Republic), the second named author was supported by Project pb96-0758 (Spain).

Copyright Clearance Centre, Inc. Serial-fee code: 0004-9727/02 \$A2.00+0.00. 
A Banach space $X$ is called weakly compactly generated, if $X$ contains a weakly compact set which is linearly dense in $X$.

The main results of this note are the following two theorems. In their proofs we shall use the following notation.

If $M$ is a bounded set in $X$, we shall say that the dual norm $\|\cdot\|$ on $X^{*}$ is $M$-locally uniformly rotund if $\sup _{x \in M}\left|\left(x^{*}-x_{n}^{*}\right)(x)\right| \rightarrow 0$ whenever $x^{*}, x_{n}^{*} \in X^{*}$ and $2\left\|x^{*}\right\|^{2}+2\left\|x_{n}^{*}\right\|^{2}-$ $\left\|x^{*}+x_{n}^{*}\right\|^{2} \rightarrow 0$. If $M=B_{X}$, we get the usual notion of local uniform rotundity (see for example [6]).

\section{THEOREM 1.}

(i) Let $M$ be a bounded subset in a Banach space $X$. Then $M$ is relatively weakly compact if and only if for every norming subspace $Y$ of $X^{*}$, there is an equivalent $Y$-lower semicontinuous norm on $X$ that is $M$-smooth.

(ii) Let $M$ be a bounded set in the dual space $X^{*}$. Then $M$ is relatively weakly compact if and only if there is an equivalent dual norm on $X^{*}$ which is $M$ smooth.

Theorem 2. Assume that $X$ is a Banach space of density $\omega_{1}$. Then $X$ is weakly compactly generated if and only if there exist a bounded linearly dense set $M$ in $X$, an equivalent norm $\|\cdot\|$ on $X$ which is $M$-smooth, and a projectional resolution of the identity $\left(P_{\alpha} ; \omega_{0} \leqslant \alpha \leqslant \omega_{1}\right)$ on $(X,\|\cdot\|)$ with $P_{\alpha}(M) \subset \overline{\operatorname{conv}}(M \cup-M)$ for every $\omega_{0} \leqslant \alpha \leqslant \omega_{1}$.

Recall that the projectional resolution $\left(P_{\alpha} ; \omega_{0} \leqslant \alpha \leqslant \omega_{1}\right)$ of the identity is a transfinite sequence of projections such that $P_{\omega_{0}}=0, P_{\omega_{1}}=$ Identity. For all $\omega_{0}<\alpha \leqslant$ $\beta \leqslant \omega_{1}$, the following hold: $\left\|P_{\alpha}\right\|=1, P_{\alpha} X$ is separable, $P_{\alpha} P_{\beta}=P_{\beta} P_{\alpha}=P_{\alpha}$, and $\bigcup_{\gamma<\alpha} P_{\gamma+1} X$ is dense in $P_{\alpha} X$. For more information on projectional resolutions of the identity we refer to [3, Chapter VI], [4, Chapter 6], or [6, Chapter 11] for example.

In order to avoid technical difficulties, we have formulated the results for spaces of density $\omega_{1}$ only.

The rest of this paper is devoted to the proofs of Theorem 1 and Theorem 2.

Let $Y$ be a subspace of the dual space $X^{*}$. We put

$$
\|x\|_{Y}=\sup \left\{x^{*}(x) ; x^{*} \in B_{X} \cdot \cap Y\right\}, \quad x \in X .
$$

Note that $\|\cdot\|_{Y}$ is lower semicontinuous with respect to the topology $w(X, Y)$ of pointwise convergence on the elements of $Y$ ( $Y$-lower semicontinuous, in short). We can check that $\|\cdot\|_{Y}$ is the largest one among all $Y$-lower semicontinuous convex minorants of the norm $\|\cdot\|$. This is why $\|\cdot\|_{Y}$ is called the $Y$-lower semicontinuous envelope of $\|\cdot\|$. Note that $B_{\left(X,\|\cdot\|_{Y}\right)}=\overline{B_{(X,\|\cdot\|)}} w(X, Y)$. If $\|\cdot\|_{Y}$ is an equivalent norm on $X$, then $Y$ is called a norming subspace of $X^{*}$. If $\|\cdot\|_{Y}=\|\cdot\|$, then $Y$ is called 1-norming. Obviously, $Y$ is 1-norming for the norm $\|\cdot\|_{Y}$. We note that a subspace $Y$ is 1-norming if and only if 
$B_{X} \cdot=\bar{B}_{X} \cdot \cap Y^{*}$, if and only if the norm $\|\cdot\|$ on $X$ is $Y$-lower semicontinuous, or if and only if $B_{X}$ is $w(X, Y)$-closed. For a bounded subset $M$ of $X$ we denote

$$
\left|x^{*}\right|_{M}=\sup \left\{\left|x^{*}(m)\right| ; m \in M\right\}, \quad x^{*} \in X^{*} .
$$

The following proposition, whose proof is standard and will be omitted, gives a Šmulyanlike characterisation of the $M$-smoothness (see for example [3, Theorem I.1.4 (i)] or [6, Lemma 8.4]).

Proposition 3. Let $(X,\|\cdot\|)$ be a Banach space, let $M$ be a bounded subset of $X$, and let $x \in S_{X}$. Then the following statements are equivalent.

(i) The norm $\|\cdot\|$ is $M$-smooth at $x$.

(ii) Whenever $\left(f_{n}\right)$ and $\left(g_{n}\right)$ are sequences in $B_{X^{*}}$ such that $f_{n}(x) \rightarrow 1$ and $g_{n}(x) \rightarrow 1$, then $\left|f_{n}-g_{n}\right|_{M} \rightarrow 0$ as $n \rightarrow \infty$.

Therefore, if the dual norm is $M$-locally uniformly rotund, then the original norm is $M$-smooth.

Proof of Theorem 1: (i) Assume that the condition holds for the set $M$. The argument we shall follow has its origin in the proof of [5, Lemma 1]. Take any $x^{* *}$ in the weak ${ }^{*}$ closure of $M$ and assume that $x^{* *} \notin X$. Then $Y:=x^{* *-1}(0)$ is a norming subspace of $X^{*}$ (see for example [6, Chapter 3]). Find $\||\cdot|||$ an equivalent $Y$-lower semicontinuous norm on $X$ that is $M$-smooth. By the Bishop-Phelps theorem, there exists $x^{*} \in S_{\left(X^{*},\|\cdot \cdot \mid\|\right)}$ and $x \in S_{(X,\|\cdot\| \cdot \|)}$ such that $\left\langle x^{* *}, x^{*}\right\rangle \neq 0$ and $\left\langle x, x^{*}\right\rangle=1$. Find a sequence $\left(y_{i}^{*}\right)$ in $B_{\left(X^{*},\|\cdot\| \|\right)} \cap Y$ such that $y_{i}^{*}(x) \stackrel{w^{*}}{\rightarrow} x^{*}(x)=1$. As $\|\cdot\| \mid \|$ is $M$-smooth, Proposition 3 gives that $\left|x^{*}-y_{i}^{*}\right|_{M} \rightarrow 0$. We recall that $x^{* *}$ is in the weak ${ }^{*}$ closure of $M$. Since the convergence of $y_{i}^{*}$ to $x^{*}$ is uniform on $M$, we thus have $x^{* *}\left(x^{*}-y_{i}^{*}\right) \rightarrow 0$. As $x^{* *}\left(y_{i}^{*}\right)=0$ for all $i \in \mathbb{N}$, we have $\left\langle x^{* *}, x^{*}\right\rangle=0$, a contradiction. Therefore the weak ${ }^{*}$ closure of $M$ belongs to $X$ and hence $M$ is relatively weakly compact.

Assume now that $M$ is relatively weakly compact. According to the Davis-FigielJohnson-Pelczyński factorisation theorem (see, for example, [4, Theorem 1.2.3] or [6, Theorem 11.17]), there exist a reflexive space $(R,|\cdot|)$ and a bounded linear operator $T: R \rightarrow X$ with $M \subset T\left(B_{R}\right)$. Following Troyanski (see, for example, [3, Section VII.1]), we may and do assume that the norm on $R^{*}$ dual to the norm $|\cdot|$ on $R$ is locally uniformly rotund. Put

$$
D=\bigcup\left\{\alpha B_{(X,\|\cdot \mid\|)}+\beta T\left(B_{(R,|\cdot|)}\right) ; \alpha \geqslant 0, \beta \geqslant 0, \alpha^{2}+\beta^{2} \leqslant 1\right\} .
$$

Then $D$ is a convex symmetric bounded and linearly dense set in $X$. Using the weak compactness of $B_{(R,|\cdot|)}$, it is not difficult to show that the set $D$ is weakly closed, and hence closed. Let $\||\cdot|\|$ be the Minkowski functional of $D$; this is an equivalent norm on $X$ and $B_{(X,\|! \cdot I\|)}=D$.

Now, let $Y$ be any norming subspace of $X^{*}$ and let $\|\cdot\|_{Y}$ and $\|\cdot \cdot\|_{Y}$ be the $Y$-lower semicontinuous envelopes of $\|\cdot\|$ and $\|\cdot\| \mid \|$ respectively. Then we have 


$$
\begin{aligned}
B_{\left(X,\|\cdot\| \cdot \|_{Y}\right)} & ={\overline{B_{(X,\|\cdot \cdot\| l)}}}^{\omega(X, Y)}=\bar{D}^{w(X, Y)} \\
& =\bigcup\left\{\alpha B_{\left(X,\|\cdot\| \cdot \|_{Y}\right)}+\beta T\left(B_{(R, H)}\right) ; \alpha \geqslant 0, \beta \geqslant 0, \alpha^{2}+\beta^{2} \leqslant 1\right\} .
\end{aligned}
$$

Here we used the weak, and hence $w(X, Y)$ compactness of the set $T\left(B_{(R,|\cdot|)}\right)$.

Having this, we get that for every $x^{*} \in X^{*}$,

$$
\begin{aligned}
\left\|x^{*}\right\|_{Y}^{2} & =\sup \left\{x^{*}(\alpha b+\beta T(r))^{2}: b \in B_{(X,\|\cdot\| Y)}, r \in B_{(R,|\cdot|)}, \alpha \geqslant 0, \beta \geqslant 0, \alpha^{2}+\beta^{2} \leqslant 1\right\} \\
& =\sup \left\{\alpha\left\|x^{*}\right\|_{Y}+\beta\left|T^{*} x^{*}\right|: \alpha \geqslant 0, \beta \geqslant 0, \alpha^{2}+\beta^{2} \leqslant 1\right\}^{2}=\left\|x^{*}\right\|_{Y}^{2}+\left|T^{*} x^{*}\right|^{2} .
\end{aligned}
$$

In order to check that $\|\cdot \cdot\|_{Y}$ is $M$-locally uniformly rotund, consider $x^{*}, x_{n}^{*} \in X^{*}$ for which

$$
2\|\| x^{*}\left\|Y_{Y}^{2}+2\right\| x_{n}^{*}\left\|_{Y}^{2}-\right\| \mid x^{*}+x_{n}^{*} \|_{Y}^{2} \rightarrow 0 \text { as } n \rightarrow \infty .
$$

Using the convexity, we get

$$
2\left|T^{*} x^{*}\right|^{2}+2\left|T^{*} x_{n}^{*}\right|^{2}-\left|T^{*} x^{*}+T^{*} x_{n}^{*}\right|^{2} \rightarrow 0 \text { as } n \rightarrow \infty .
$$

Since the norm $|\cdot|$ on $R^{*}$ is locally uniformly rotund, we conclude that $\left|T^{*} x_{n}^{*}-T^{*} x^{*}\right| \rightarrow 0$, that is, $\sup \left\{\left(x_{n}^{*}-x^{*}\right)(x) ; x \in T\left(B_{R}\right)\right\} \rightarrow 0$ as $n \rightarrow \infty$. Now it remains to recall that $M \subset T\left(B_{R}\right)$.

(ii) Assume $M \subset X^{*}$ is relatively weakly compact. By Theorem 1 (i) there is an equivalent dual norm norm on $X^{*}$ that is $M$-smooth. In order to see this, it suffices to note that $X$ is a norming subspace of $X^{* *}$.

On the other hand, assume that the norm of $X^{*}$, dual to the norm $\|\cdot\|$ of $X$, is $M$ smooth. Assume that $M$ is not relatively weakly compact. Like in the proof of Theorem 1 (i), there exists $x^{* * *}$ in the weak ${ }^{*}$ closure of $M$ which does not belong to $X^{*}$. Denote by $x^{*}$ the restriction of $x^{* * *}$ to $X$. Consider $x^{*}$ as an element of $X^{* * *}$. We need to show that $F:=x^{* * *}-x^{*}=0$. Assume this is not the case and choose an element $x^{* *} \in S_{X}$ *. with $F\left(x^{* *}\right) \neq 0$ and $x^{* *}\left(y^{*}\right)=1$ for some $y^{*} \in S_{X}$. Find a net $\left(y_{\iota}\right)$ in $B_{X}$ such that $y_{\iota} \rightarrow x^{* *}$ in the weak* topology. As the dual norm is $M$-smooth, $\left|x^{* *}-y_{\iota}\right|_{M} \rightarrow 0$. The element $x^{* * *}$ belongs to the weak ${ }^{*}$ closure of $M$. Thus $x^{* * *}\left(x^{* *}-y_{\iota}\right) \rightarrow 0$. As $x^{*} \in X^{*}$, we have $x^{*}\left(x^{* *}-y_{\iota}\right) \rightarrow 0$. Thus $F\left(x^{* *}-y_{\iota}\right) \rightarrow 0$. However, $F\left(y_{\iota}\right)=0$ for all $\iota$. Hence $F\left(x^{* *}\right)=0$, a contradiction. This finishes the proof of Theorem 1 .

In the proof of Theorem 1, some ideas from [1] were used.

In the proof of Theorem 2 we shall use the following definition.

Definition: Let $(X,\|\cdot\|)$ be a Banach space. Let $M$ be a bounded linearly dense subset of $X$. We shall say that a projectional resolution of the identity $\left(P_{\alpha} ; \omega_{0} \leqslant \alpha \leqslant \mu\right)$ 
on $(X,\|\cdot\|)$ is $M$-shrinking if $P_{\alpha}(M) \subset \overline{\mathrm{conv}}(M \cup-M)$ and

$$
P_{\alpha}^{*}\left(X^{*}\right)={\overline{\bigcup_{\beta<\alpha} P_{\beta+1}^{*}\left(X^{*}\right)}}^{\left.1 \cdot\right|_{M}}
$$

for every $\omega_{0}<\alpha \leqslant \mu$.

If $M=B_{X}$, we get the usual notion of a shrinking projectional resolution of the identity (see for example $[3,4,6]$ ). It follows that a projectional resolution of the identity $\left(P_{\alpha}\right)$ is $M$-shrinking if and only if for every $\alpha$ we have $P_{\alpha}(M) \subset \overline{c o n v}(M \cup-M)$ and $\left|P_{\beta}^{*} x^{*}-P_{\alpha}^{*} x^{*}\right|_{M} \rightarrow 0$ as $\beta \uparrow \alpha$ for every $x^{*} \in X^{*}$. Note that the Mackey-Arens theorem (see for example [6, Theorem 4.33]) implies that, if $M$ is a weakly compact set in a Banach space $X$ and $\left(P_{\alpha}\right)$ is a projectional resolution of the identity on $X$ such that $P_{\alpha}(M) \subset \overline{c o n v}(M \cup-M)$ for every $\alpha$, then $\left(P_{\alpha}\right)$ is $M$-shrinking.

Lemma 4. Let $(X,\|\cdot\|)$ be a Banach space whose norm is $M$-smooth for some bounded set $M \subset X$. Assume that $\left(P_{\alpha} ; \omega_{0} \leqslant \alpha \leqslant \mu\right)$ is a projectional resolution of the identity on $(X,\|\cdot\|)$ that satisfies $P_{\alpha} M \subset \overline{c o n v}(M U-M)$ for every $\alpha$. Then $\left(P_{\alpha}\right)$ is $M$-shrinking.

Proof of Lemma 4: We need to prove that

$$
P_{\alpha}^{*}\left(X^{*}\right)=\left.{\overline{\bigcup_{\beta<\alpha} P_{\beta+1}^{*}\left(X^{*}\right)}}^{1} \cdot\right|_{M}
$$

for every $\omega_{0}<\alpha \leqslant \mu$. Fix such $\alpha$. It is enough to prove the inclusion " $C$ ". Fix $x^{*} \in P_{\alpha}^{*}\left(X^{*}\right)$. We note that $P_{\beta}^{*} x^{*} \rightarrow P_{\alpha}^{*} x^{*}$ in the weak star topology as $\beta \uparrow \alpha$. Assume first that $\left\|P_{\alpha}^{*} x^{*}\right\|=P_{\alpha}^{*} x^{*}(x)$ for some $x \in S_{X}$. As $\left\|P_{\beta}^{*} x^{*}\right\|=\left\|P_{\beta}^{*} P_{\alpha}^{*} x^{*}\right\| \leqslant\left\|P_{\alpha}^{*} \dot{x}^{*}\right\|$, Proposition 3 guarantees that $\left|P_{\beta}^{*} x^{*}-x^{*}\right|_{M}$ as $\beta \uparrow \alpha$. Hence $x^{*}$ belongs to the right hand side of the above formula.

Second, assume that $x^{*}$ is not norm attaining. Then the Bishop-Phelps theorem and the canonical isometry between $\left(P_{\alpha} X\right)^{*}$ and $P_{\alpha}^{*}\left(X^{*}\right)$ enable us to find a norm attaining $y^{*} \in P_{\alpha}^{*} X^{*}$ such that $\left\|x^{*}-y^{*}\right\|<\varepsilon$, where $\varepsilon>0$ is an arbitrary, a priori given positive number. Then, by the first case, $\left|P_{\beta}^{*} y^{*}-y^{*}\right|_{M} \rightarrow 0$ as $\beta \uparrow \alpha$. This yields that $\limsup \left|P_{\beta}^{*} x^{*}-x^{*}\right|_{M} \leqslant 2 \varepsilon$. Here we used that $P_{\beta}(M) \subset \overline{\mathrm{conv}}(M \cup-M)$. As $\varepsilon>0$ was $\beta+\alpha$ arbitrary, we get that $x^{*}$ belongs to the right hand side of the above formula.

Proof of Theorem 2: Sufficiency. Assume we have $\|\cdot\|, M$ and $\left(P_{\alpha} ; \omega_{0} \leqslant \alpha\right.$ $\left.\leqslant \omega_{1}\right)$ as in the statement. For every $\omega \leqslant \alpha<\omega_{1}$ we find a countable dense set $\left\{m_{i}^{\alpha} ; i \in \mathbb{N}\right\}$ in $\left(P_{\alpha+1}-P_{\alpha}\right) M \cap B_{X}$. Put

$$
C=\left\{\frac{1}{i} m_{i}^{\alpha} ; \omega_{0} \leqslant \alpha<\omega_{1}, i \in \mathbb{N}\right\} \cup\{0\} .
$$


The set $C$ is linearly dense in $X$. It remains to prove that $C$ is weakly compact. Let $\left(c_{j}\right)_{j \in \mathbf{N}}$ be a sequence of disctinct elements in the set $C$. According to the EberleinŚmulyan theorem, it is enough to prove that this sequence has a weakly convergent subsequence. For $j \in \mathbb{N}$ find $\omega_{0} \leqslant \alpha_{j}<\omega_{1}$ and $i_{j} \in \mathbb{N}$ such that $c_{j}=\left(1 / i_{j}\right) m_{i_{j}}^{\alpha_{j}}$. If the set $\left\{i_{j} ; j \in \mathbb{N}\right\}$ is infinite, then it is easy to find a subsequence of $\left(c_{i}\right)$ which converges to 0 (even in norm). Assume now that the set $\left\{i_{j} ; j \in \mathbb{N}\right\}$ is finite. Then $\left\{\alpha_{j} ; j \in \mathbb{N}\right\}$ is an infinite set. By passing to a subsequence, if necessary, we may, and do assume that $\alpha_{1}<\alpha_{2}<\cdots$. Let $Y$ denote the linear span of the set $\bigcup_{\omega_{0} \leqslant \alpha<\omega_{1}}\left(P_{\alpha+1}^{*}-P_{\alpha}^{*}\right) X^{*}$. Because of the "orthogonality" of the projections $P_{\alpha+1}-P_{\alpha}$, we can see that for every $x^{*} \in Y$ we have $x^{*}\left(c_{j}\right)=0$ for all $j \in \mathbb{N}$ large enough. Using Lemma 4, we can prove by transfinite induction that $\bar{Y}^{\left.\left.\right|^{\mid}\right|_{M}}=X^{*}$. Thus $x^{*}\left(c_{j}\right) \rightarrow 0$ for every $x^{*} \in X^{*}$ and the weak compactness of the set $C$ follows. Therefore $X$ is weakly compactly generated.

Necessity. Assume that $X$ is weakly compactly generated. Then there exists a linearly dense and weakly compact set $M$ in $X$. By Theorem 1 (i), $X$ admits an equivalent norm $\|\cdot\|$ that is $M$-smooth. As $X$ is weakly compactly generated, there is a projectional resolution of identity $\left(P_{\alpha}\right)$ such that $P_{\alpha}(M) \subset \overline{c o n v}(M \cup-M)$ for each $\alpha$ (see for example $[3,4,6]$.) For the sake of completeness we shall show the argument here. For $n \in \mathbb{N}$, let $\|\cdot\|_{n}$ be the Minkowski functional of the set $\overline{\operatorname{conv}}(M \cup-M)+B_{X} / n$. As in [4, p. 109], we construct on $X$ a projectional resolution of the identity $\left(P_{\alpha} ; \omega_{0} \leqslant \alpha \leqslant \omega_{1}\right)$ such that $\left\|P_{\alpha}\right\|_{n}=1$ for every $n \in \mathbb{N}$ and every $\alpha>\omega_{0}$. Then

$$
P_{\alpha}(M) \subset P_{\alpha}\left(\overline{\operatorname{conv}}(M \cup-M)+\frac{1}{n} B_{X}\right) \subset \overline{\operatorname{conv}}(M \cup-M)+\frac{1}{n} B_{X}
$$

for every $n \in \mathbb{N}$, and hence $P_{\alpha}(M) \subset \overline{\operatorname{conv}}(M \cup-M)$. This finishes the proof of Theorem 2 .

If the norm of a Banach space is $M$-smooth, then $M$ is an Asplund set ([1], see [4, Section 1.4] for the definition). Then, using [12] or [14], one can prove the following corollary. However in this note we present a simpler proof of Corollary 5. A Banach space $X$ is called weakly Lindelöf determined if its dual unit ball in its weak star topology is a Corson compact. A compact space $K$ is a Corson compact if $K$ is homeomorphic to a subset $S$ of some $[-1,+1]^{\Gamma}$ in its pointwise topology such that all elements of $S$ are countably supported in $[-1,+1]^{\Gamma}$. Every subspace of a weakly compactly generated space is weakly Lindelöf determined (see for example [4]). For more on weakly Lindelöf determined spaces see for example $[4,6]$ and references therein.

COROLlary 5. Assume that $X$ is a weakly Lindelöf determined Banach space of density $\omega_{1}$. Then $X$ is weakly compactly generated if and only if $X$ admits an equivalent $M$-smooth norm for some bounded and linearly dense subset $M$ of $X$.

PROOF: The necessity follows immediately from Theorem 2.

Assume that the condition holds. Let $\|\cdot\|$ be the equivalent $M$-smooth norm on $X$. 
The space $X$ admits a projectional resolution of the identity $\left(P_{\alpha}\right)$ such that $P_{\alpha}(M) \subset$ $\overline{\operatorname{conv}}(M \cup-M)$ for all $\alpha$ (see for example [4, p. 109]). Hence $X$ is weakly compactly generated by Theorem 2 .

Remarks. Theorem 1 (i) does not hold true if the condition on the $Y$-lower semicontinuity is dropped. In order to see this, take any nonreflexive space $X$ with Fréchet smooth norm and put $M:=B_{X}$ (see for example [3, Chapter 2] or [6, Chapter 8]).

Theorem 1 (i) should be compared with the following result in [8]. If $X$ is a subspace of a weakly compactly generated space and $Y$ is a norming subspace in $X^{*}$, then there is an equivalent norm on $X$ that is Gâteaux smooth and $Y$-lower semicontinuous.

Theorem 1 (ii) should be compared with Corollary III-8 in [2], which asserts that $X^{*}$ is weakly compactly generated if $X$ is an Asplund space and $X^{*}$ admits an equivalent dual Gâteaux dfferentiable norm.

Hajek proved in [9] that the James tree space $J T$ admits an equivalent norm whose dual norm $\|\cdot\|$ is Gâteaux smooth. As $J T^{*}$ is not even a subspace of a weakly compactly generated space (see for example [6, Chapter 11]), Theorem 1(ii) shows that the norm $\|\cdot\|$ on $J T^{*}$ is not $M$-smooth for any bounded linearly dense set $M$ in $X^{*}$.

Theorem 2 generalises the classical result that the space is reflexive if the norm $X^{*}$ dual to the norm of $X$ is Fréchet smooth (see for example [6, p. 244]).

Note that the conditions in Corollary 5 are satisfied if $X$ is a subspace of a weakly compactly generated space of density $\omega_{1}$ having a Fréchet differentiable norm. This is the main result in [11] that is discussed in [3, Chapter 6], [4, Chapter 8] or [6, Chapter 11] for example.

While the non weakly compactly generated space $C\left[0, \omega_{1}\right]$ of continuous functions on the ordinal segment admits an equivalent $C^{\infty}$ smooth norm ([10]), this space admits no Gâteaux smooth norm that would be either a lattice norm ([7]) or pointwise lower semicontinuous for $t \in\left[0, \omega_{1}\right)([8])$. Note that every equivalent norm on $C\left[0, \omega_{1}\right]$ is pointwise lower semicontinuous as $\left[0, \omega_{1}\right]$ is a scattered space (see for example $[6$, Theorem 12.28]).

\section{REFERENCES}

[1] J.M. Borwein, 'Weak local supportability and application to approximations', Pacific J. Math 82 (1979), 323-338.

[2] R. Deville and G. Godefroy, 'Some applications of projective resolutions of identity', Bull. London Math. Soc. 67 (1993), 183-199.

[3] R. Deville, G. Godefroy and V. Zizler, Smoothness and renormings in Banach spaces, Pitman Monographs and Surveys in Pure and Applied Mathematics 64 (Longman Scientific and Technical, Harlow, 1993).

[4] M. Fabian, Gâteaux differentiability of convex functions and topology. Weak Asplund spaces (John Wiley and Sons, New York, 1997). 
[5] M. Fabian, G. Godefroy and V. Zizler, 'The structure of uniformly Gâteaux smooth Banach spaces', Israel J. Math. 124 (2001), 243-252.

[6] M. Fabian, P. Habala, P. Hájek, J. Pelant, V. Montesinos and V. Zizler, Functional analysis and infinite dimensional geometry, CMS Books in Mathematics 8 (Springer-Verlag, New York, 2001).

[7] M. Fabian, P. Hájek and V. Zizler, 'A note on lattice renormings', Comment. Math. Univ. Carolin. 38 (1997), 263-272.

[8] M. Fabian, V. Montesinos and V. Zizler, 'Pointwise lower semicontinuous smooth norms', Arch. Math. (to appear).

[9] P. Hájek, 'Dual renormings of Banach spaces', Comment. Math. Univ. Carolin. 37 (1996), 241-253.

[10] R. Haydon, 'Trees in renorming theory', Proc. London Math. Soc. 78 (1999), 541-584.

[11] K. John and V. Zizler, 'Smoothness and its equivalents in weakly compactly generated Banach spaces', J. Funct. Anal. 15 (1974), 161-166.

[12] J. Orihuela, W. Schachermayerand M. Valdivia, 'Every Radon-Nikodým Corson compact is Eberlein compact', Studia Math. 98 (1991), 157-174.

[13] H.P. Rosenthal, 'The heredity problem for weakly compactly generated Banach spaces', Compisito. Math. 28 (1974), 83-111.

[14] Ch. Stegall, 'More facts about conjugate Banach spaces with the Radon-Nikodým property II', Acta Univ. Carolin. Math. Phys. 32 (1991), 47-57.

Mathematical Institute of the Czech Academy of Sciences

Žitná 25, 11567

Prague 1

Czech Republic

e-mail: fabian@math.cas.cz

Department of Mathematical Sciences

University of Alberta

632 Central Academic Building

Edmonton, Alberta T6G 2G1

Canada

e-mail: vzizler@math.ualberta.ca
Departamento de Matemática Aplicada

E.T.S.I. Telecomunicación

Universidad Politécnica de Valencia

C/Vera, s/n. 46071

Valencia

Spain

e-mail: vmontesinos@mat.upv.es 